\title{
DIY goes in vivo
}

\author{
When existing systems don't get the job done, a growing community of researchers are customizing-and \\ sharing-their own solutions.
}

\section{Jim Kling}

A $s$ a graduate student at MIT in 2010, Josh Siegle was investigating the mechanisms of learning and memory in mice. He wanted to implant wire electrodes to detect changes in the electrical potentials produced by neurons and to carry out experiments involving closedloop feedback. In a closed-loop experiment, "rather than just having the brain do whatever it does and measure the output, you perturb the brain based on what's happening inside of it. That's an incredibly powerful way of studying a system, because you can explore the parameter space much more quickly when your perturbations respond to activity in the brain itself, rather than just happening at random times," says Siegle, who is now a researcher at the Allen Institute for Brain Science.

One experiment he wanted to try required stimulating a mouse's hippocampus only during the peaks of an electrical wave recorded inside of this brain region. Other scientists had proposed that electrical waves in the hippocampus help distinguish information that is being stored from information that is being remembered. If the stimulation occurred while the mouse was exploring a maze, it could enhance the animal's ability to store and later recall the memory of the location of a reward. This had the potential to explain how the brain differentiates newly-forming memories from those already stored. Such an experiment "would be impossible without closed-loop feedback. Stimulating the hippocampus at random times would not have allowed us to test this particular hypothesis," says Siegle.

The trouble was that commercial data acquisition systems were ill-equipped to handle such experiments, not to mention expensive. The precise circuitry required to amplify and digitize these signals cost about $\$ 100,000$. That hardware was available to Siegle, but it may have been prohibitively expensive for others trying to replicate his work. Meanwhile, the available open-source alternatives were poorly documented or relied on outdated hardware. Together with his MIT colleague Jakob Voigts, Siegle built the system they needed for their own lab's internal use.

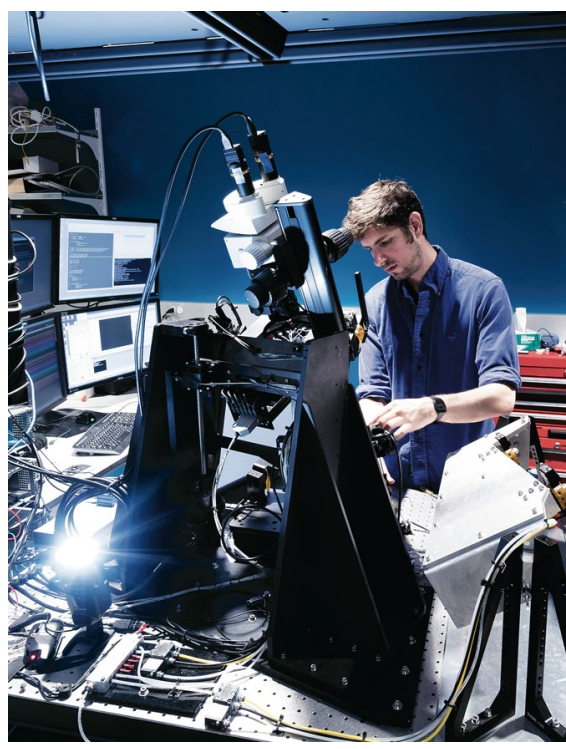

DIY man: Josh Siegle has been building with his own software and hardware since his graduate days. With Open Ephys, he wants to help others do the same. Credit: E. Dinnel

When they ran the experiment with their customized equipment, they found that they could enhance spatial navigation in freely moving mice by activating specific cells at different phases of the electrical wave. The work ultimately suggests that encoding and retrieval of information may be differentiated by the phase of the electrical wave at which each process occurs.

Siegle and Voigts quickly realized that the tools they had built would be useful for others as well and their collaboration evolved into Open Ephys (http://www. open-ephys.org/), a non-profit that now coordinates the development and distribution of open-source hardware and software for electrophysiology experiments.

The open-source software they developed took inspiration from digital audio workstations for music production, which include numerous plugins that can produce a wide range of tones and effects. "Users can mix and match which plugins they want to use to achieve a particular sound. We wanted to do the same thing for neuroscience," says Siegle.

Open Ephys software can accommodate user-developed plugins that import data from external hardware, create visualizations, deliver closed-loop feedback to the brain, and perform other tasks. "Our software is ultimately independent of the hardware people are using to acquire their data. So if you built a particular algorithm for closed-loop feedback, or an algorithm for data visualization, you could use that for any data input, rather than having to rebuild the software from scratch," says Siegle.

In addition to software, Open Ephys continues to supply open-source hardware designs for things like implants and headstages that scientists can build themselves. They also began to manufacture and sell hardware in order to make it even more accessible. Lisbon-based OEPS (https://www.open-ephys.org/store) now handles hardware distribution for the organization, and the profits pay the stipend of a support person for Open Ephys software. "Our main goal is to do great science, and we want to make it as easy as possible for everyone else to do great science as well," says Siegle.

That goal is reinforced by the modularity of the software they produce. "Anyone can take a plugin that was built by someone halfway around the world and integrate that into their data acquisition pipeline the next day," says Siegle.

Siegle notes that there are two target audiences for Open Ephys. One is researchers who simply can't afford the commercial tools required to do electrophysiology. The other is researchers who want to do cutting edge science that commercial systems, however reliable, lack the flexibility to achieve. "Having tools that are affordable and open source will help on both ends of that spectrum," says Siegle.

\section{Tools of the trade}

Siegle's desire to help others succeed underpins much of the open-source movement in electrophysiology and behavioral research. Traditionally, neuroscientists have relied on commercial 


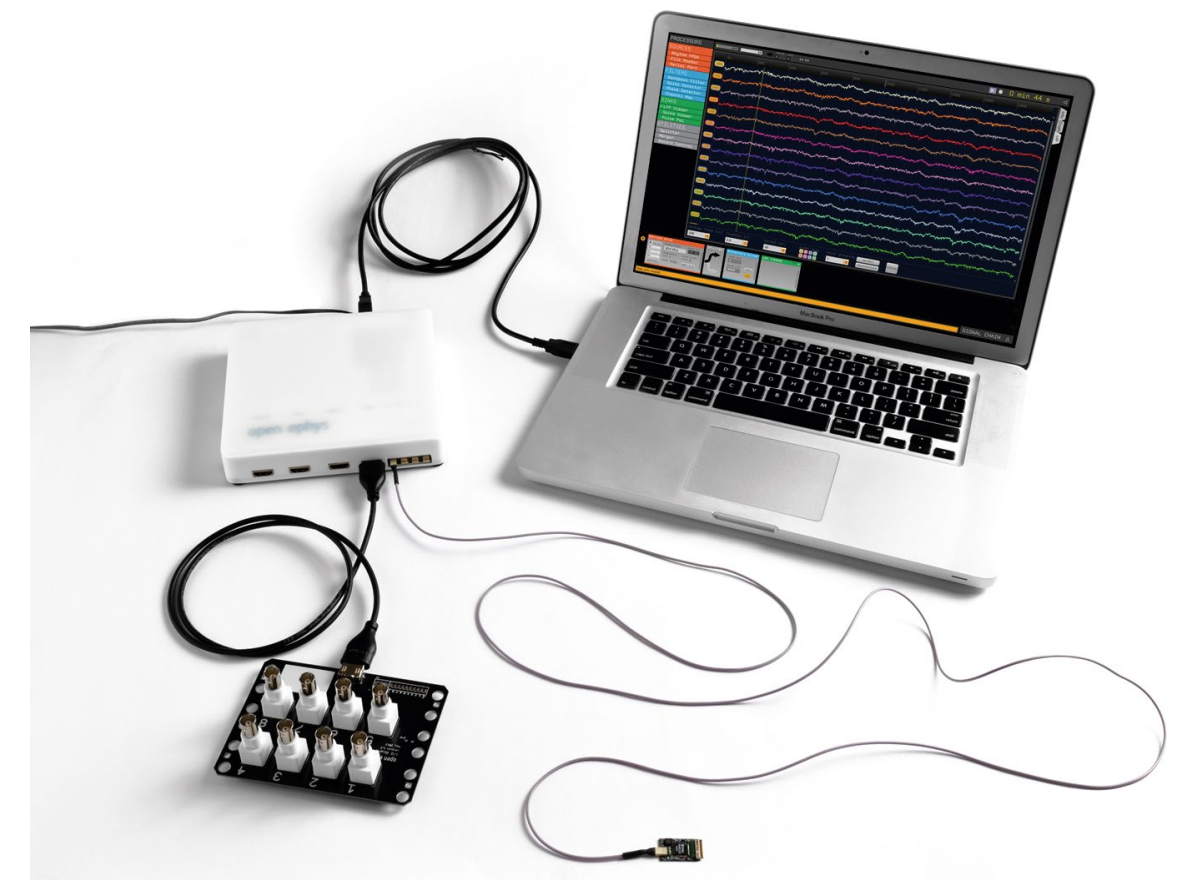

A simple set up: The Open Ephys acquisition board, shown with Intan headstage and laptop. Credit: J. Henkel. Reprinted with permission from Siegle et al. J Neural Eng 14, 045003 (2017)

systems for the tools to conduct their research, but technological innovations and new tools have put much of the development power into the hands of researchers themselves.

Siegle realized the data acquisition hardware he needed could be greatly simplified by turning to integrated amplifier chips from Intan Technologies (http://www.intantech.com/), which would also reduce the overall system cost to under $\$ 10,000$. Intan was founded in 2010 by Reid Harrison, a professor of electrical engineering who had a keen interest in neuroscience. "I quickly realized that there was a need for miniaturized electronics, because most of the electronics used to detect the electrical signals in the brain and nerves was very antiquated. It was really 1980s technology. People were still using large, rack-mounted boxes that were very expensive, very large and bulky," says Harrison.

The new chip weighs less than a gram and can sit directly on an animal's brain, where it amplifies, digitizes, and processes data from probes that make direct contact within individual neurons. Only one, very thin cable is required to deliver digital data to a computer for analysis. Miniaturization reduced the cost of the technology by replacing traditional circuit components and eliminating the large circuit board area required for them, and the software that runs it is free. "This has opened up our technology to researchers in regions of the world that maybe don't have as large a research budget as people in the United States or in Europe," says Harrison, who is president and CEO of Intan.

Intan's chips are great at acquiring signals, but home-grown systems almost always require a microprocessor or a microcomputer to control automated functions, such as temperature control or time stamping. Arduino microprocessors (https://www.arduino.cc) can perform such simple tasks. Raspberry Pi microcomputers (https://www.raspberrypi.org) can be used to control the microprocessors, as well as conduct data analysis and other more demanding tasks. Introduced in 2012, the Raspberry Pi costs about 100 euros (US \$120). It was first developed for education purposes and for developing countries as an alternative to full scale computers, but it soon found use among do-it-yourselfers, who employed it for home entertainment systems, video games, and a range of other uses. Then researchers caught drift of it.

Open source has been central to these devices since their beginning. "The Italians who originally shared (Arduino) with the web provided so many details that it was really easy for people to pick up this little microcontroller and use it for all different kinds of things, including very complex (tasks)," says Joshua Pearce, a professor of materials science and engineering at Michigan Technological University and author of The Open-source Lab: How to Build Your Own Hardware and Reduce Research Costs.

Another key tool is $3 \mathrm{D}$ printing, which has been around for decades but has only recently become broadly available. That's because $3 \mathrm{D}$ printing was itself made open source. "The reason you've heard about $3 \mathrm{D}$ printing is that it went open source and dropped the cost down to where anyone can get them," said Pearce.

Combine those advances with opensource software and designs readily sharable through Open Ephys and similar networks, and you have the makings for big strides in DIY projects.

\section{The open-source fly habitat}

Giorgio Gilestro combined several of these tools to create the ethoscope for studying fly behavior. The ethoscope is a $3 \mathrm{D}$-printable machine powered by a Raspberry $\mathrm{Pi}$ microcomputer, all at a cost of about 100 euros (US \$120).

Gilestro, a lecturer in systems biology at Imperial College London, is investigating the mechanisms that govern sleep and wakefulness, and his model of choice is the fruit fly. His research initially relied on an infrared beam to register a fly's movement when it crossed its path. But that didn't work when the fly was moving the other direction. Gilestro designed the ethoscope to solve that problem. His wasn't the first such system, but it was the first to be made open source.

In October 2017, Gilestro's team published a paper in PLoS Biology outlining plans for producing a $10 \mathrm{~cm} \times 13 \mathrm{~cm} \times 19 \mathrm{~cm}$ ethoscope using 3D printing, folded cardboard, or, more whimsically, Lego blocks (technical drawings for 3D printing and assembly and full list of components: https://lab.gilest.ro/ethoscope). The latter two options are geared towards educational applications.

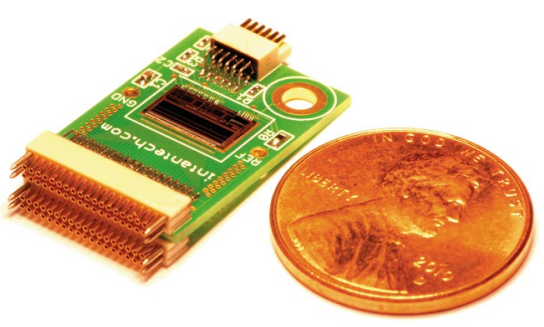

Penny for your thoughts? Intan's 64-channel amplifier board. Credit:R. Harrison, Intan Technologies 
In a typical experiment, researchers place multiple ethoscopes into a climatecontrolled chamber. Each ethoscope is powered by a USB cable and communicates through WiFi with a desktop computer that collects and stores data. A researcher can operate any number of ethoscopes simultaneously. The main limitation is that the onboard Raspberry Pi microcomputers generate significant heat, requiring that they be placed in a climate controlled environment. Gilestro's solution: modified commercial wine coolers with temperature control provided by Arduino microprocessors.

Because different experiments may demand unique arenas, Gilestro's team has developed example arenas for long-term uses such as sleep or longevity analyses, and short-term experiments investigating decision-making or courtship. The video of the animal can be tracked in real time, with the animal's position and angle recorded at about four frames per second, or the video can be analyzed later if faster time resolution is needed (up to $60 \mathrm{hz}$ ).

Gilestro's approach could also help improve throughput, which has long been a problem in animal behavior studies. Researchers want to compare many animals in different conditions. For example, a sleep researcher studying jet lag might want to study dozens of flies simultaneously, each living in a different simulated time zone. But experimental setups like these can be costly, bulky, and difficult to scale up. The ethoscope's low cost and simple modularity makes multi-animal studies more accessible.

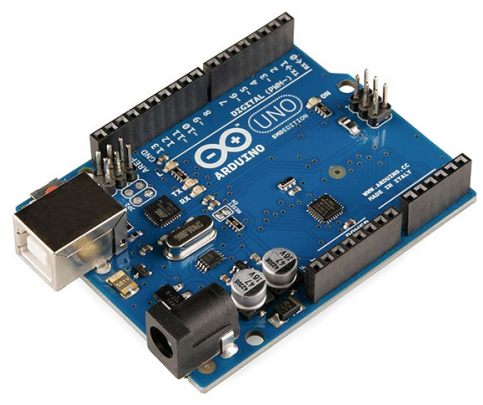

Staples gone micro: Arduino microprocessors (pictured) and Raspberry PI microcomputers are inexpensive hardware options. Credit: SparkFun Electronics

In one experiment, Gilestro's team analyzed 1,400 flies at once, using 20 wine coolers, each housing three to four ethoscopes. Like Siegle, Gilestro's team conducts closed feedback loop experiments. They expose the flies to food, or to odors they like or don't like-sometimes that means the same chemical compound, just in different concentrations - and then record how they react. "This second dimension of interacting with flies is really powerful," says Gilestro.

\section{New frontiers}

Open-source hardware and software can drive research forward in unexpected paths. Commercial products tend to be reliable and consistent, but they often lack flexibility.

André Maia Chagas, a researcher in Thomas Euler's laboratory at The Centre for Integrative Neuroscience (Tübingen, Germany), employs open-source hardware
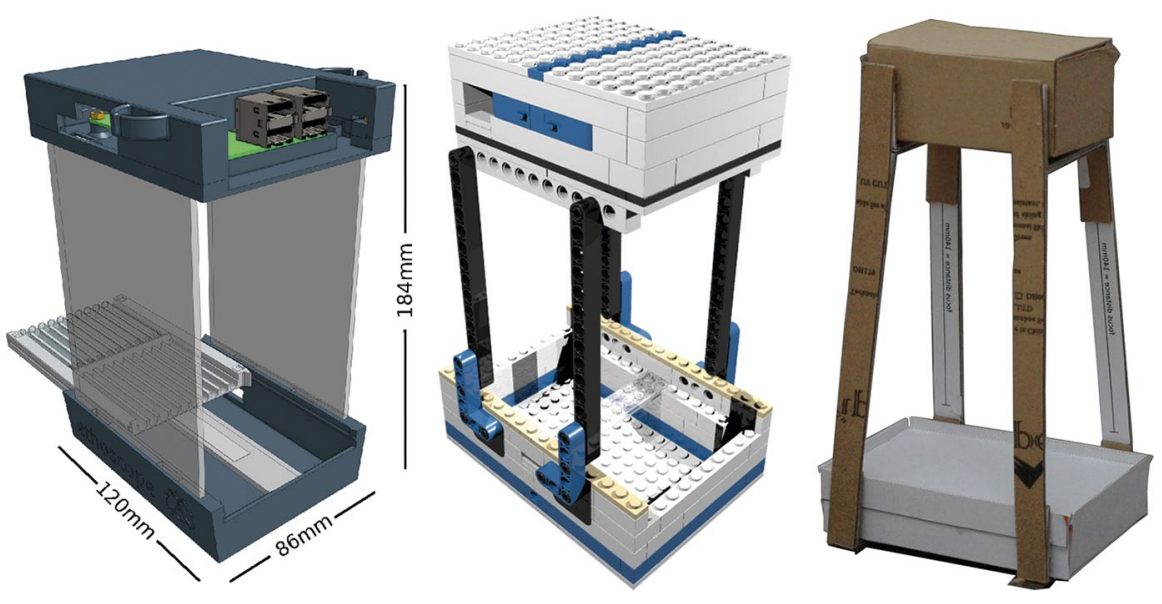

Take your pick: The chassis that holds Giorgio Gilestro's ethoscope comes in a variety of finishes: 3D-printed plastic (left), Lego (middle), or paper and cardboard (right). Adapted with permission from Geissmann et al. PLoS Biol 15, e2003026 (2017).

to customize experimental setups. The lab he works in images mice retinas to investigate the first stages of vision. To do that, he needs to see how different cell types respond to light stimulation, and that requires rapid collection of images from the top to the bottom of the retina. Chagas attempted this with a commercial driver that used electrical current to alter the shape of a lens, which in turn changed the focus plane and individual cell being recorded. But the commercial system was too slow to change the imaging plane to keep up with the experimental protocol.

Chagas' team reached out to another lab that had created an open-source driver for the lens, built a copy for themselves, and found it to be effective. It turned out the commercial driver was trying to do many things at once, and those calculations slowed the system down. "The open-source system was much simpler, and that made it work much better," says Chagas. And the cost? About 50 euros, compared to 4-500 for the commercial system. "You go to Radio Shack and you buy a couple of resistors, a couple of electronic components, and you put this thing together," he says.

Experiences like that have changed his approach to research. "Whenever I have a problem in the lab, instead of finding a solution on my own right away, I always look for open-source solutions online before going and reinventing the wheel," says Chagas.

In fact, altering one researcher's design, as Chagas did, is a central tenet of the open-source movement. A researcher develops equipment, and another expands it to find new uses. "What I have found with all kinds of devices that I have put out there is that people will make improvements on them for their own experiments, and when I go back to do another experiment, I can start where they left off. By sharing my work, it becomes better. It's like having an international team of engineers constantly hammering on your project," says Pearce.

But the DIY, open-source movement in electrophysiology and animal behavior research isn't just saving money and expanding research options. It's also affecting how researchers view their own tools. "A lot of times people have this idea that I have this machine, I put my sample in, I press a button, and I have to believe whatever comes out. Now, because you're building your own equipment and learning a little bit about electronics, a little bit about design... If something comes out that doesn't 


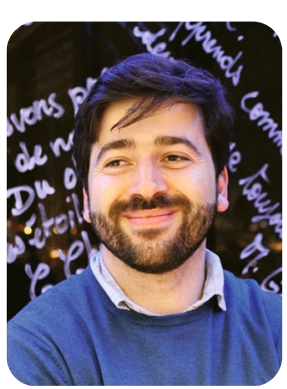

"Whenever I have a

problem in the lab,

instead of finding a

solution on my own right

away, I always look for

open-source solutions

online before going and

reinventing the wheel,"

says André Maia Chagas.

Image: Makery make sense, you know if there is a problem, what the problem is, and how to fix it. More importantly, you know how the process of analysis works," says Chagas.

The approach does demand something extra from researchers. Siegle says that members of Open Ephys are almost all self-taught engineers, and he recognizes that not all neuroscientists are eager to delve into that arena. Still, "it's a lot easier than it appears. It's not that difficult to do basic things. The tools for engineering are designed to be easy for people to understand. So I encourage all scientists to not be daunted by building your own tools," he says.

Jim Kling

Freelance writer, Bellingham, Washington, USA. e-mail: jkling@gmail.com

Published online: 23 May 2018

https://doi.org/10.1038/s41684-018-0066-Z

\section{PLAS LABS INC..}

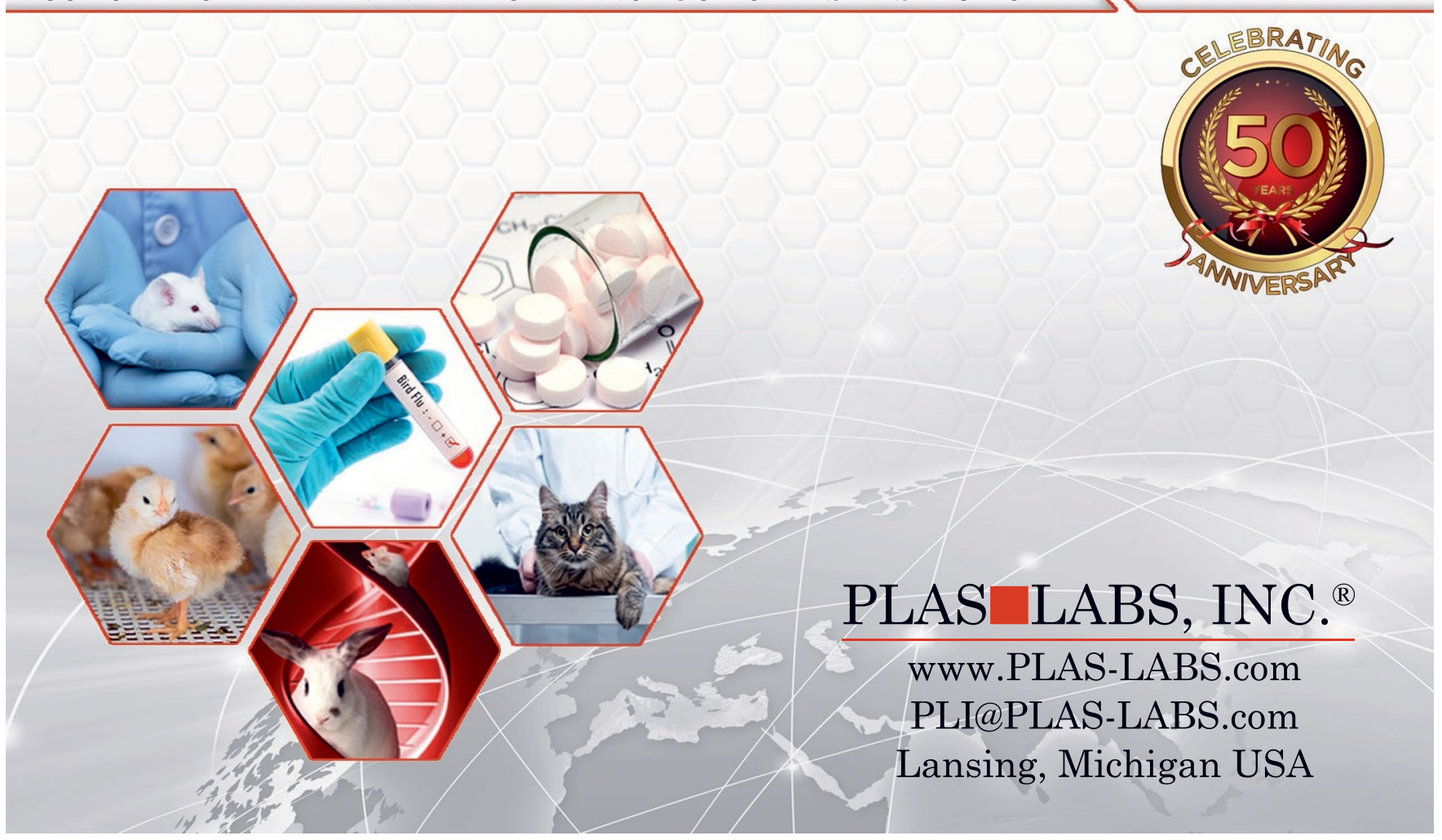

